Supporting Information

\title{
Electrostatic Jet Engineering of Flexible Composite Pressure Sensors for Physical Applications
}

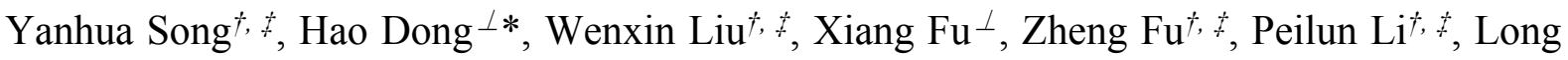

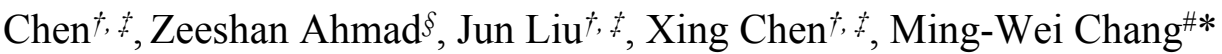

${ }^{\dagger}$ Key Laboratory for Biomedical Engineering of Education Ministry of China Zhejiang University, Hangzhou 310027, P. R. China

Zhejiang Provincial Key Laboratory of Cardio-Cerebral Vascular Detection Technology and Medical Effectiveness Appraisal, Zhejiang University, 310027, China

${ }^{\perp}$ Research Perception Research Institute, Zhejiang Lab, Hangzhou 311100, China

$\S$ De Montfort University, The Gateway, Leicester, LE1 9BH, UK

\# Nanotechnology and Integrated Bioengineering Centre, University of Ulster, Jordanstown Campus, Newtownabbey, Northern Ireland, BT37 0QB, UK

\section{Corresponding Author}

*Email: cnhaodong@zhejianglab.com; m.chang@ulster.ac.uk 
Table S1 Comparison of different 3DP sensors and FPS.

\begin{tabular}{llllc}
\hline Active materials & $\begin{array}{l}\text { Mechanical } \\
\text { property }\end{array}$ & $\begin{array}{l}\text { Measurement range } \\
(\mathrm{Pa})\end{array}$ & $\begin{array}{l}\text { Maximum } \\
\text { sensitivity }\end{array}$ & Ref. \\
\hline SWCNTs/PDMS microstructure & Flexible & $0.6-1.2 \mathrm{k}$ & $1.8 \mathrm{kPa}^{-1}$ & 9 \\
Graphene oxide (GO)/Paper & Flexible & $0-20 \mathrm{k}$ & $17.2 \mathrm{kPa}^{-1}$ & 10 \\
rGO micropyramid & Flexible & $1.5-1.4 \mathrm{k}$ & $5.5 \mathrm{kPa}^{-1}$ & 11 \\
Graphene/PDMS & Flexible & $1-12 \mathrm{k}$ & $8.5 \mathrm{kPa}^{-1}$ & 12 \\
Graphene /PU sponge & Flexible & $9-10 \mathrm{k}$ & $0.26 \mathrm{kPa}^{-1}$ & 13 \\
Graphene/PDMS & Flexible & $/$ & $15.9 \mathrm{kPa}^{-1}$ & 14 \\
CNT/ Graphene & Flexible & $0-5.8 \mathrm{k}$ & $19.8 \mathrm{kPa}^{-1}$ & 15 \\
This work & Flexible & $\mathbf{0 - 3 . 4 ~ k}$ & $\mathbf{2 5 . 9} \mathbf{k P a}^{-1}$ & \\
\hline
\end{tabular}

Table S2 Thickness and bending radius of the device for the bending strain test.

\begin{tabular}{ccc}
\hline Thickness of sample $(\mathrm{d})$ & Bending radius $(\mathrm{r}) \times 2$ & Bending strain $(\varepsilon=\mathrm{d} / 2 \mathrm{r} \times 100 \%)$ \\
\hline $0.45 \mathrm{~mm}$ & $3.6 \mathrm{~mm}$ & $12.5 \%$ \\
$0.45 \mathrm{~mm}$ & $3.6 \mathrm{~mm}$ & $12.5 \%$ \\
$0.45 \mathrm{~mm}$ & $2.7 \mathrm{~mm}$ & $16.7 \%$ \\
$0.45 \mathrm{~mm}$ & $2.6 \mathrm{~mm}$ & $17.3 \%$ \\
$0.45 \mathrm{~mm}$ & $2.4 \mathrm{~mm}$ & $18.8 \%$ \\
$0.45 \mathrm{~mm}$ & $2.2 \mathrm{~mm}$ & $20.5 \%$ \\
$0.45 \mathrm{~mm}$ & $2.0 \mathrm{~mm}$ & $22.5 \%$ \\
$0.45 \mathrm{~mm}$ & $1.8 \mathrm{~mm}$ & $25.0 \%$ \\
$0.45 \mathrm{~mm}$ & $1.6 \mathrm{~mm}$ & $28.1 \%$ \\
$0.45 \mathrm{~mm}$ & $1.5 \mathrm{~mm}$ & $30.0 \%$ \\
$0.45 \mathrm{~mm}$ & $1.3 \mathrm{~mm}$ & $34.6 \%$ \\
$0.45 \mathrm{~mm}$ & $1.1 \mathrm{~mm}$ & $40.9 \%$ \\
$0.45 \mathrm{~mm}$ & $0.9 \mathrm{~mm}$ & $50.0 \%$ \\
\hline
\end{tabular}




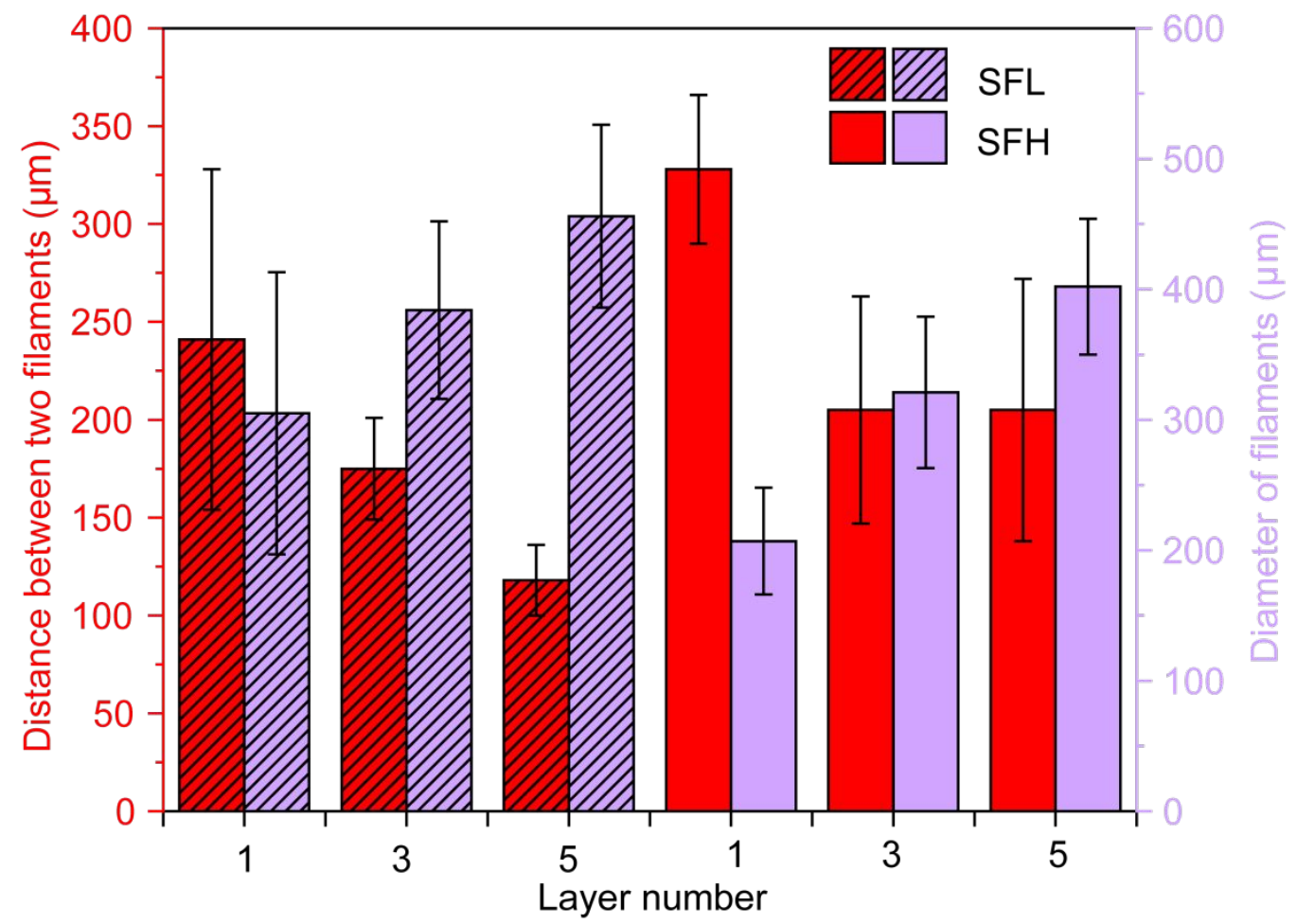

Figure S1 Size distribution of jetted layers from 1 to 5 layers for SFH and SFL. 

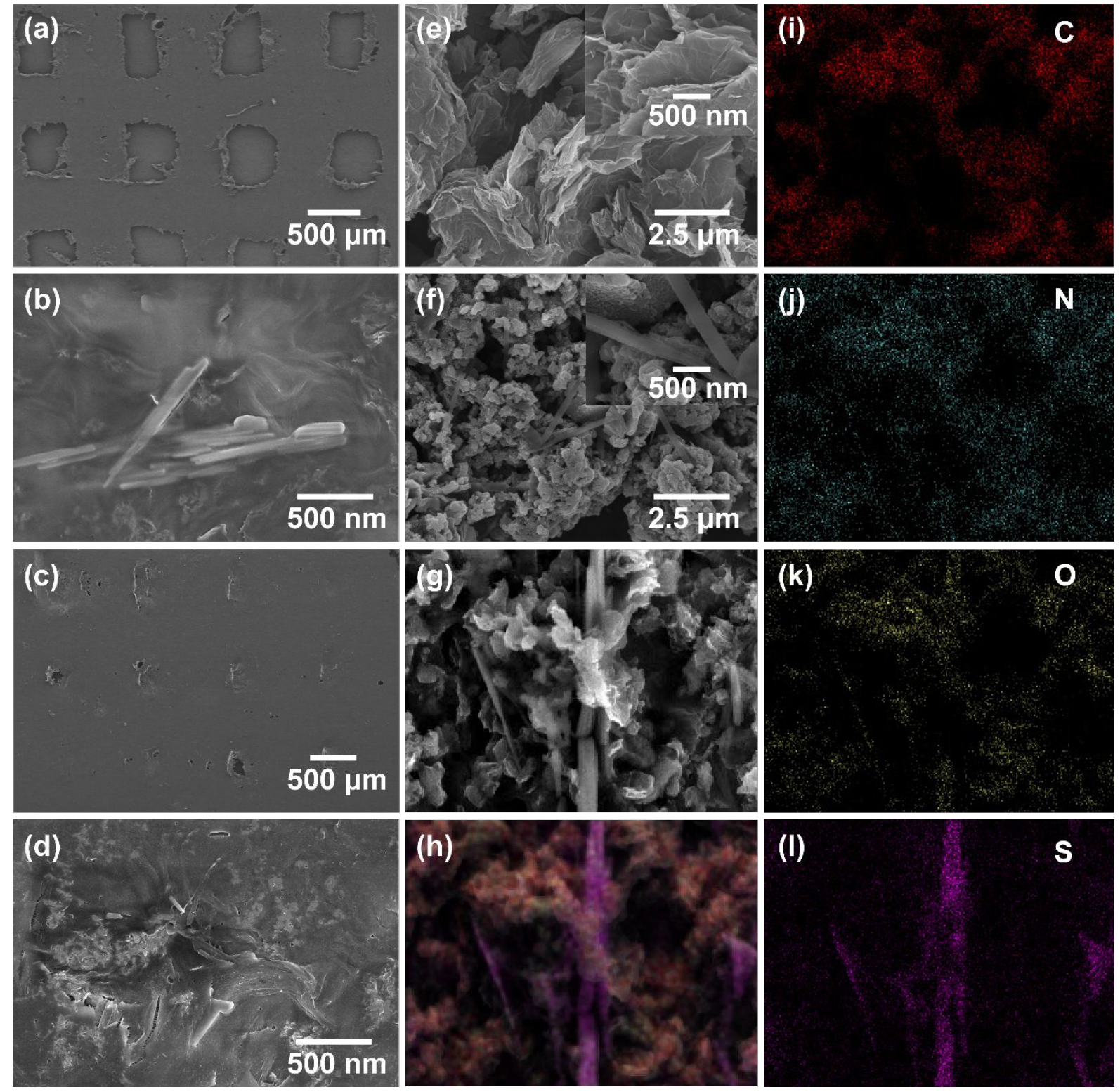

Figure S2 Electron micrographs and EDS results of jetted SFH and SFL (five layers). Electron micrographs of SFH $(\mathbf{a}, \mathbf{b})$ and SFL $(\mathbf{c}, \mathbf{d})$ collected on the bottom surfaces at different magnifications. Electron micrographs of G (e), PANI (f), and PEO/PANI/G sensing layer (g). EDS mapping of chemical elements: PEO/PANI/G sensing layer showing elements (h), C (i), $\mathrm{N}(\mathbf{j}), \mathrm{O}(\mathbf{k})$, and $\mathrm{S}(\mathbf{l})$. 


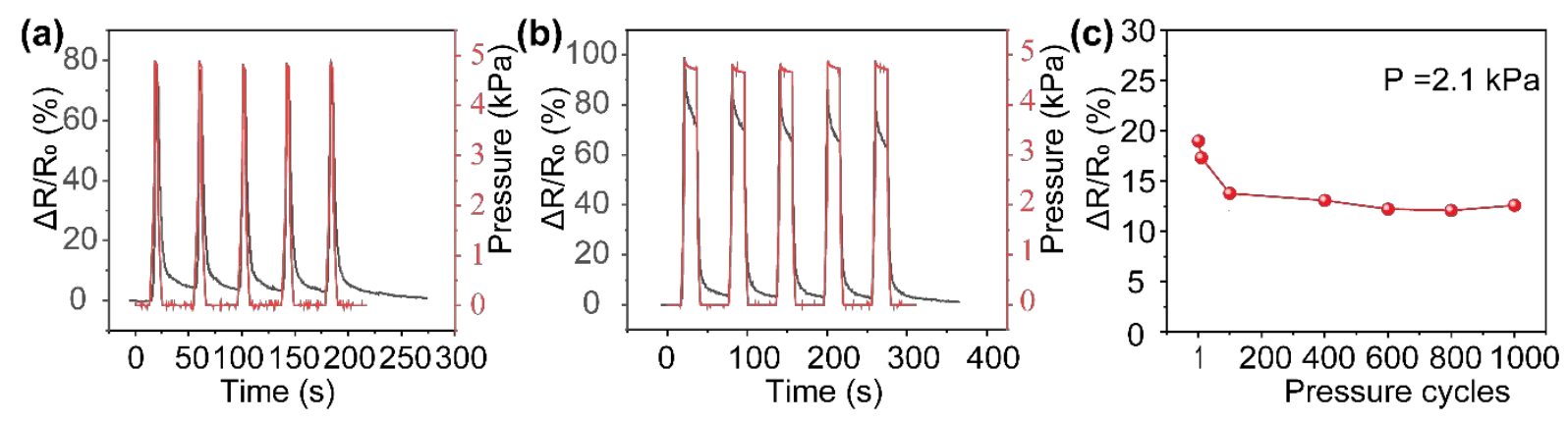

Figure S3 Mechanoelectrical tests of the FPS. Time dependence of relative resistance change at different dwell times $(\mathbf{a}, \mathbf{b})$. Here the test machine stopped at the highest point in air without exerting pressure. (c) Relative resistance variation versus loading-unloading pressure cycles under an applied pressure of $2.1 \mathrm{kPa}$.
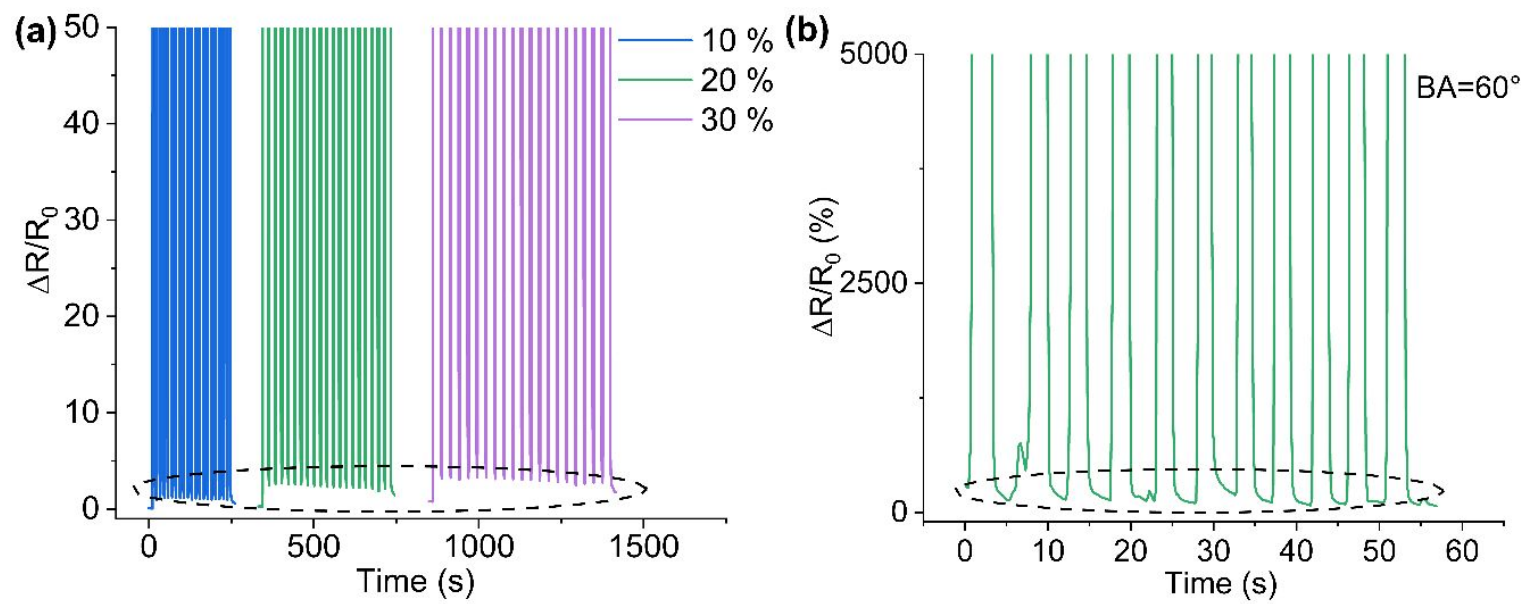

Figure S4 (a) Enlarged portion of curve showing relative resistance variation versus time with increasing tensile strain. (b) Enlarged portion of curve showing relative resistance variation versus time at a finger bending angle of $60^{\circ}$. 

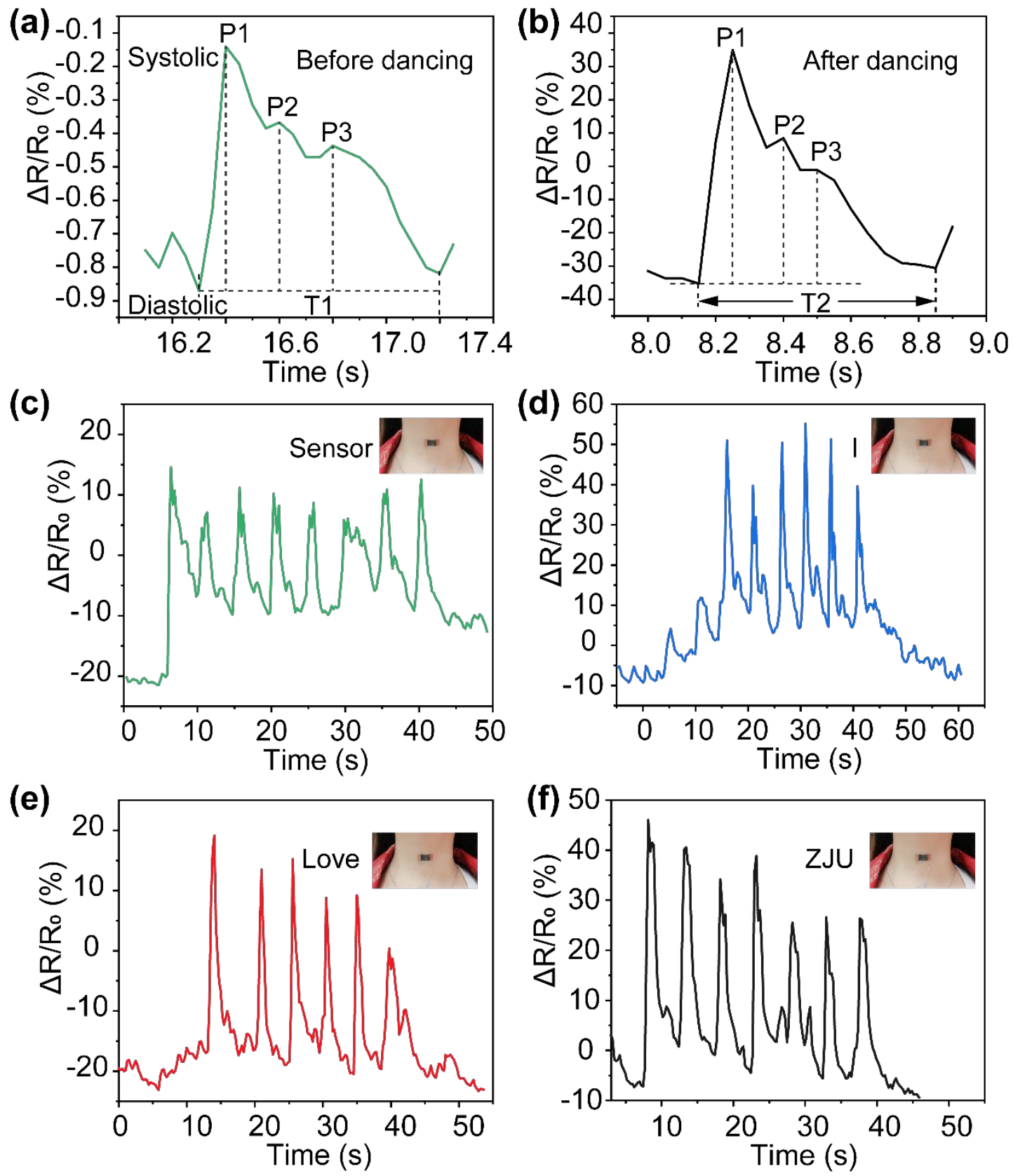

Figure S5 Three wave components before (a) and after dancing (b): percussion wave (P1), tidal wave (P2) and diastolic wave (P3) of typical pulse waves. (c)-(f) output signals of repeatable words of "sensor", "I", “love”, and "ZJU”, respectively. 